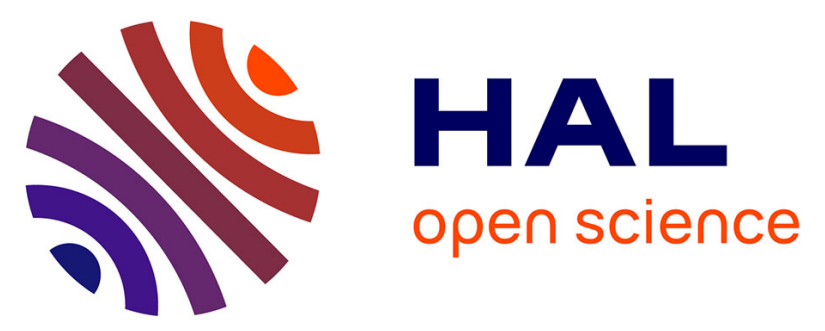

\title{
Extension of Bilbro-McMillan Charge Density Wave-Superconductivity coexistence relation to quantum régimes: application to superconducting domes around quantum critical points
}

\author{
Manuel Nunez-Regueiro
}

\section{To cite this version:}

Manuel Nunez-Regueiro. Extension of Bilbro-McMillan Charge Density Wave-Superconductivity coexistence relation to quantum régimes: application to superconducting domes around quantum critical points. Journal of Magnetism and Magnetic Materials, 2015, 376, pp.25-28. 10.1016/j.jmmm.2014.04.036 . hal-00977931

\section{HAL Id: hal-00977931 \\ https://hal.science/hal-00977931}

Submitted on 23 May 2014

HAL is a multi-disciplinary open access archive for the deposit and dissemination of scientific research documents, whether they are published or not. The documents may come from teaching and research institutions in France or abroad, or from public or private research centers.
L'archive ouverte pluridisciplinaire HAL, est destinée au dépôt et à la diffusion de documents scientifiques de niveau recherche, publiés ou non, émanant des établissements d'enseignement et de recherche français ou étrangers, des laboratoires publics ou privés. 
Extension of Bilbro-McMillan Charge Density WaveSuperconductivity coexistence relation to quantum régimes: application to superconducting domes around quantum critical points

\author{
Manuel Núñez-Regueiro \\ Institut Néel, Université Grenoble Alpes \&Centre Nationale de la Recherche \\ Scientifique, BP166, cedex 09, 38042 Grenoble, France
}

Quantum critical points (QCP) accompanied by superconductivity are ubiquitous in condensed matter physics. In general, the transition temperature $T_{O}$ of an ordered state, e.g. antiferromagnetic, goes to zero under the influence of an external parameter, e.g. pressure. Superconductivity appears before the disappearance of the ordered state, but reaches its maximum $T_{c}$ when $T_{O}=0$. Presently, the implications of the QCP's on superconductivity are a subject of debate. It is propose here that both transition temperatures satisfy the relation $\widetilde{T}_{O}{ }^{2}+\widetilde{T}_{c}{ }^{2}=1$, where the tilde indicates normalization to the maximum values. Inspired from the basic postulate of $S O(N)$ theories of superconductors, it is proposed as an extension from Bilbro-McMillan relation. 
The study of phase transitions is central to the understanding of the physical properties of condensed matter [1]. Standard phase transitions, at a transition temperature $T_{c} \neq 0$, can be treated classically, even those between different quantum states. Quantum phase transitions, where the transition between two different phases takes place at $T_{c}=0$, are qualitatively different, as their critical fluctuations must be treated quantum mechanically [2,3]. Great progress has been made in their understanding, e.g. in the case of magnetic quantum phase transitions $[4,5,6]$. In a large number of materials including pnictides, heavy fermions, charge or spin density wave materials, the phase transition at $T_{c}=0$ as a function of a certain parameter $\delta$ (pressure, doping, etc.) from an ordered, generally bad conductor, state to a conducting disordered phase, is surrounded in the $T-\delta$ plane by a superconducting region (see Fig. 1). The central point is that there is a region where the ordered and the superconducting state (SC) coexist. For charge density waves (CDW) this coexistence has been studied by several groups $[7,8,9]$. In particular, Bilbro and McMillan (BM)[8] studied the interaction of CDW and SC in A15 materials within mean field. Here, both states compete for developing their respective gaps in the same Fermi surface. They obtained the following relation between $T_{c}$, the superconducting transition temperature, and $T_{O}$, the CDW transition temperature,

$$
T_{O}{ }^{1-n_{0}}(P) T_{c}^{n_{0}}(P)=T_{c_{M a x}}
$$

where $n_{0}$ is the fraction of the Fermi surface under the CDW gap and $T_{c_{M a x}}$ the superconducting transition with no CDW.

Different works have shown that under pressure there are clearly two regimes for the evolution of $T_{0}$ on its way to the QCP. First a classical BCS at low pressures one and then a quantum fluctuations (QF) one near the QCP. For example, both the 
one dimensional transition metal trichalcogenide $\mathrm{NbSe}_{3}$, a well-studied charge density wave (CDW) compound [10,11,12] and $\mathrm{Cr}$ metal [13] display, at low pressures, an decrease of $T_{0}$ following an exponential with pressure BCS tuned variation, consequence of the dependence of $T_{O}$ with the coupling parameter $\lambda$, i.e. $T_{O} \sim e^{-1 / \lambda}$. A quantum region appears below a crossover value of $T_{0}$ where $\mathrm{QF}$ take control through a power law behavior with an exponent $\psi=1 / 2$ (See the behavior of $\mathrm{Ag}_{0.33} \mathrm{~V}_{2} \mathrm{O}_{5}$ on Fig. 1).

It is clear that Eq. (1) can only be applied in the BCS régime and is that not valid in the $\mathrm{QF}$ region. However, at least for $\mathrm{CDW}$ and SDW materials, a direct relation between $T_{0}$ and $T_{c}$ describing that both states are the result of the same Fermi surface and electron-phonon interaction is expected and needed. In order to have a hint towards it, an analysis of how the BM relation works experimentally is clarifying.

In A-15 compounds, $n_{0} \sim$ some 10\%'s and changes only a few percents in the measured pressure range [8]. Considering then $n_{0}$ approximately constant in the pressure range, expression (1) becomes,

$$
\left(1-n_{0}\right) \ln T_{c}(P)+n_{0} \ln T_{O}(P) \approx \ln T_{c_{M a x}}
$$

We see from Fig. 2 where the variation with pressure of both transition temperatures for the two most studied A-15 compounds, $V_{3} \mathrm{Si}$ and $\mathrm{Nb}_{3} \mathrm{Sn}$ is plotted as logarithms, that expression (2) is verified, $n_{0}$ being constant within experimental error, and 0.26 for $V_{3} S i$ and 0.14 for $\mathrm{Nb}_{3} S n$; the obtained $T_{c_{M a x}}$ are $17.7 \mathrm{~K}$ and $20.1 \mathrm{~K}$ for $V_{3} S i$ and $N b_{3} S n$, respectively. Experimentally we $\ln T_{x}(P)=\ln T_{x}(0)+\alpha_{x} P$ have , $\alpha_{x}$ being a constant and $x$ either $c$ or 0 . The logarithmic variation with pressure of both transition temperatures is the result of the exponentially tuned BCS expression. In other words, at all the shown pressures, both transitions without any doubt obey BCS statistics. 
However, as described above, the CDW/SDW transition deviates from the exponentially tuned BCS behavior towards a power law dependence of the type $T_{O} \propto\left[\frac{\left(\delta_{c}-\delta\right)}{\delta_{c}}\right]^{1 / 2}$ near to the QCP. It is apparent that the transition temperature does not more follow BCS statistics, but another possibly due to the QF near the QCP. QF accelerate the rhythm of decrease of $T_{0}$, destabilizing the CDW/SDW and, at the same time, inducing a faster transfer of carriers from the CDW/SDW gap to the SC gap. Equation (1) is thus no longer valid. Phenomenologically, expression (1) adds the variations of the $T_{x}{ }^{\prime} s$ using the functional that is linear with pressure, which in the BCS régime is the logarithm of the transition temperature. The same approach may be taken for the power law régime. Thus, instead of the logarithms, as now $\left[T_{O} / T_{O}(0)\right]^{2}=1-P / P_{C}$, the squares of the transition temperatures should be added and equated to a constant. It is thus expected that the equivalent of BM for the power law region would be a summation of squares of the type

$$
a T_{O}^{2}+b T_{c}^{2}=\kappa
$$

To advance further in obtaining a BM formula for the quantum régime, it is useful to take into account the $\mathrm{SO}(5)$ formulation. In the paper [14] on $\mathrm{SO}(5)$, it was argued that the simplest way to construct an unified theory of antiferromagnetism and superconductivity for cuprates was to define a five dimensional spinor composed by the three components of the magnetization and the real and imaginary part of the dwave superconducting gap. In this way, it would be possible to recover all the relevant physics of the problem of high temperatures superconducting cuprates[15]. More complicated problems would require higher dimensions spinors to obtain all the physics, i.e. the complete phase diagram with all the possible ground states, e.g. $\mathrm{SO}(8)$ for a two chains model of correlated fermions[16]. However, experimentalists can be less ambitious, and just try to describe phenomenologically with the most 
compact expression a restricted region of the phase diagram, e.g. the one near to QCP. Basically, there are two coexisting phases. The ordered phase should rigorously have several components. However, systems with presumed different number of components, show the same behavior near the QCP. $\mathrm{SO}(5)$ and $\mathrm{SO}(4)$ has been successfully used to explain properties of the Co doped $B a F e_{2} A s_{2}$ system[17], and the Beechgard salt[18] $(T M T T F)_{2} P F_{6}$, respectively. Thus, just one component for the ordered phase may be enough for this portion of the phase diagram. A similar reasoning can be used for the superconducting phase, that may also be accounted by one component. The actual formula is suggested by expression (3). It is (Fig. 3a)

$$
\frac{T_{c}(P)^{2}}{T_{c_{M a x}}^{2}}+\frac{T_{O}(P)^{2}}{T_{O_{\text {Max }}}^{2}}=1
$$

The procedure to apply this formula is as following : the $T_{O}$ dependence with pressure, concentration, etc. is fitted by a $T_{O}(\delta)=T_{O_{M A X}}\left|\frac{\delta-\delta_{c}}{\delta_{c}}\right|^{\psi}$ (5) law, from which the dependence of $T_{C}(\delta)=T_{c_{M A X}} \sqrt{1-\left|\frac{T_{O}(\delta)}{T_{O}\left(\delta_{c_{2}}\right)}\right|^{2}}$ (6) can be calculated. The normalization to $T_{O}\left(\delta_{c_{2}}\right)$ is a way to apply the formula only to the actual region of coexistence of the ordered and superconducting phase (Fig. 3b). Thus, the dependence of $T_{O}(\delta)$ determines that of $T_{C}(\delta)$. The satisfactory application of the formula is also shown for the examples on Fig. 1.

For $\delta>\delta_{c}$ the dependence of $T_{O}$ is naturally extended to negative values (low-right quadrant of Fig. 3a), and its module is used in the determination of $T_{c}$. In the case of magnetic QCP's, this extension can be associated to the crossover or coherence line, below which the system recovers normal Fermi liquid properties. It follows the power law $T_{c o h} \sim\left(\left(\delta-\delta_{c}\right) / \delta_{c}\right)^{v z}$ [6], where $v$ is the correlation length exponent and $z$ the 
dynamical exponent. As for low dimensions $v z=\psi$ is often valid, i.e. both $T_{O} \equiv T_{N}$ and $T_{c o h}$ should follow the same power law. On the other hand, for CDW-s, a negative $T_{O}$ can be traced to the soft phonon that exists even with values of $\delta$ that hinder the occurrence of the lattice distortion[19,20]. In other words, the interaction, responsible for the ordering, does not obviously disappears when $T_{O}=0$, and the extrapolation to negatives values of $T_{O}$ is an approximation to its magnitude.

In fact, already a study of the antiferromagnetic QCP, considering that superconductivity is due to spin fluctuations caused by its criticality, arrives at a formula of similar nature by derivation of the lines of constant coherence lengths [21] $T_{c}{ }^{2}+\left|\frac{\delta-\delta_{c}}{\delta_{c}}\right|^{2}=T_{c_{\max }}{ }^{2}(7)$. In the case of a $T_{N}\left(T_{c o h}\right)$ with a critical exponent $\psi=1(v z=1)$, expressions (4) and (7) are almost identical. It is clear that the type of analysis done on Ref. 21 can be a way for the understanding of superconducting domes around QCP's of different nature.

In conclusion, a formula equivalent to the Bilbro-McMillan expression for coexistence between a charge density wave and superconductivity but applicable to the quantum fluctuations controlled regions of the phase diagram is proposed to describe the superconducting domes that appear around quantum critical points. It is shown that it describes phenomenologically the observed behavior for several systems. 

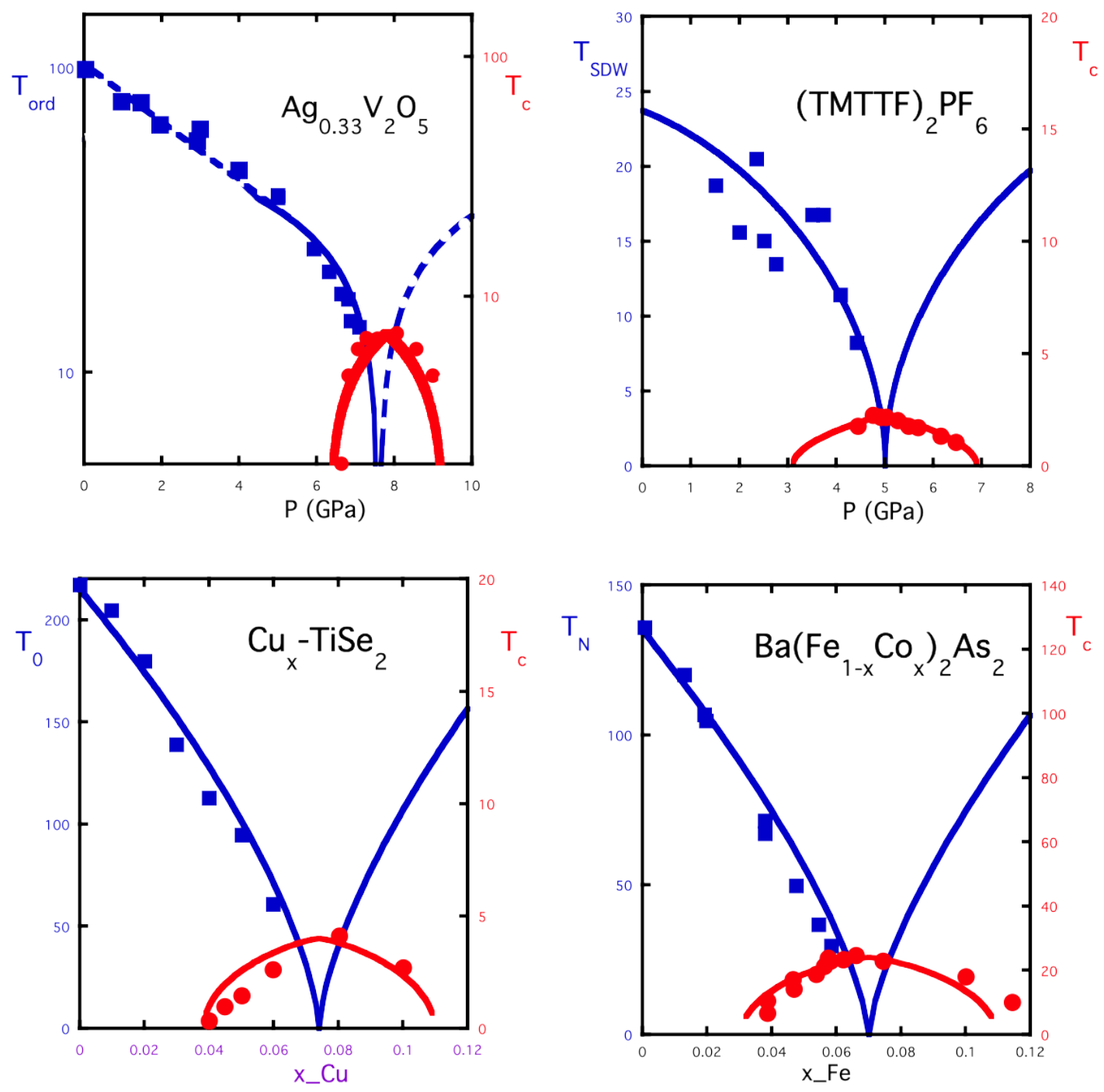

Figure 1

Examples of materials showing a QCP surrounded by a superconducting dome. The compound $\left(\mathrm{Ag}_{0.33} \mathrm{~V}_{2} \mathrm{O}_{5}\right)$ [22] follows at low pressures an exponential with pressure BCS tuned variation up to $\sim 5 \mathrm{GPa}$ (blue dashed right line). Above this pressure, the compound crossovers to a quantum fluctuations regime, following the power law of the type $T_{O} \propto\left[\frac{\left(\delta_{c}-P\right)}{\delta_{c}}\right]^{1 / 2}$ (blue solid line). The other three compounds (TMTTF $)_{2} P F_{6}$ [23], $\mathrm{Cu}_{x} \mathrm{TiSe}_{2}$ [24] and $\mathrm{Ba}\left(\mathrm{Fe}_{1-x} \mathrm{Co}_{x}\right)_{2} \mathrm{As}_{2}$ [25] show power laws at all pressures. Also shown (red circles) is the superconducting dome. The red line is obtained using formula (4), see text and Fig. 3. 


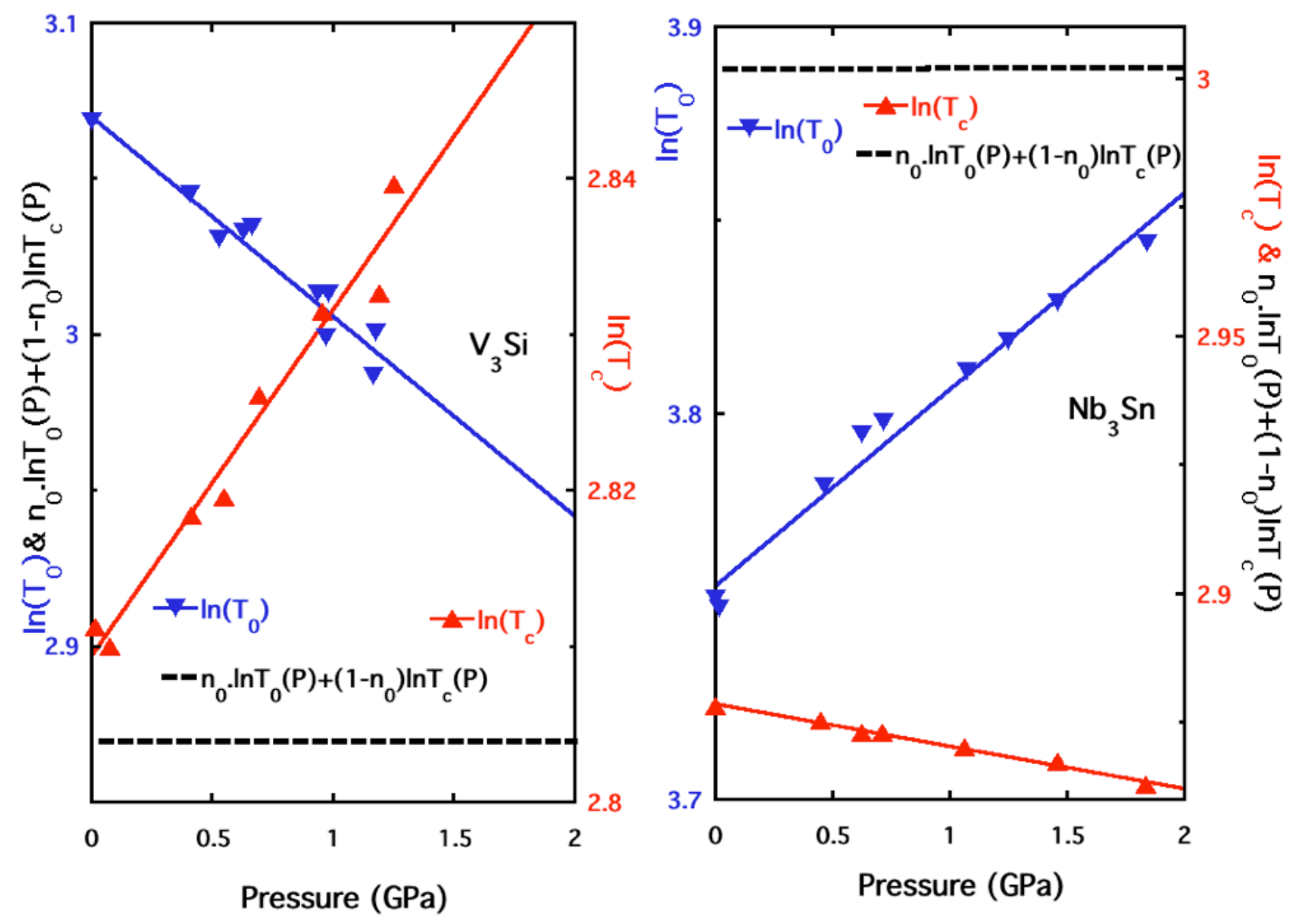

\section{Figure 2}

Left (Right) Panel: Evolution of the $\ln T_{0}$, inverted triangles, and $\ln T_{c}$, triangles, with pressure for $V_{3} \mathrm{Si}$; data from Ref. $26\left(\mathrm{Nb}_{3} \mathrm{Sn}\right.$; data from Ref. 27$)$. The straight dashed line verifies Eq. (2) with $n_{0}=0.26$ and $T_{c_{M a x}}=17.7 \mathrm{~K} \quad\left(n_{0}=0.14\right.$ and $T_{c_{M a x}}$ $=20.1 \mathrm{~K})$. 


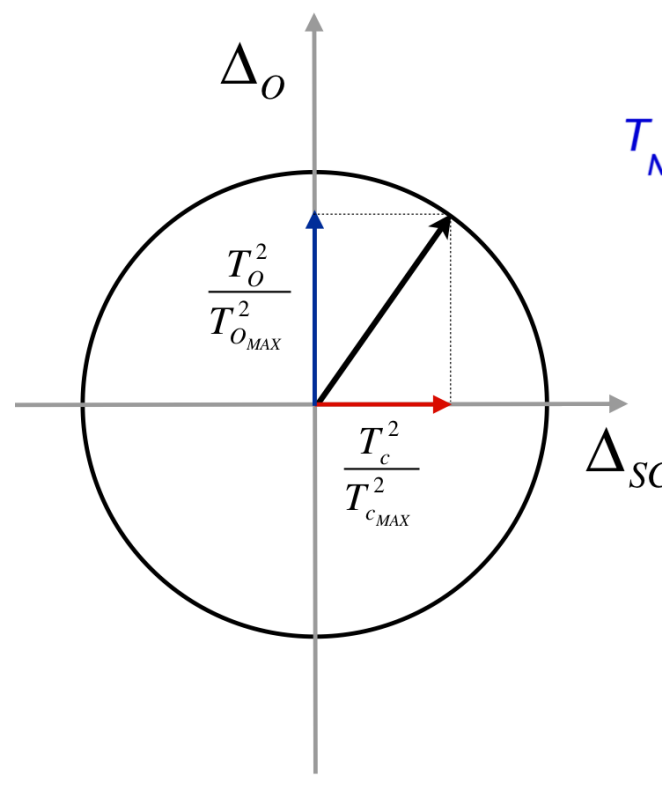

a

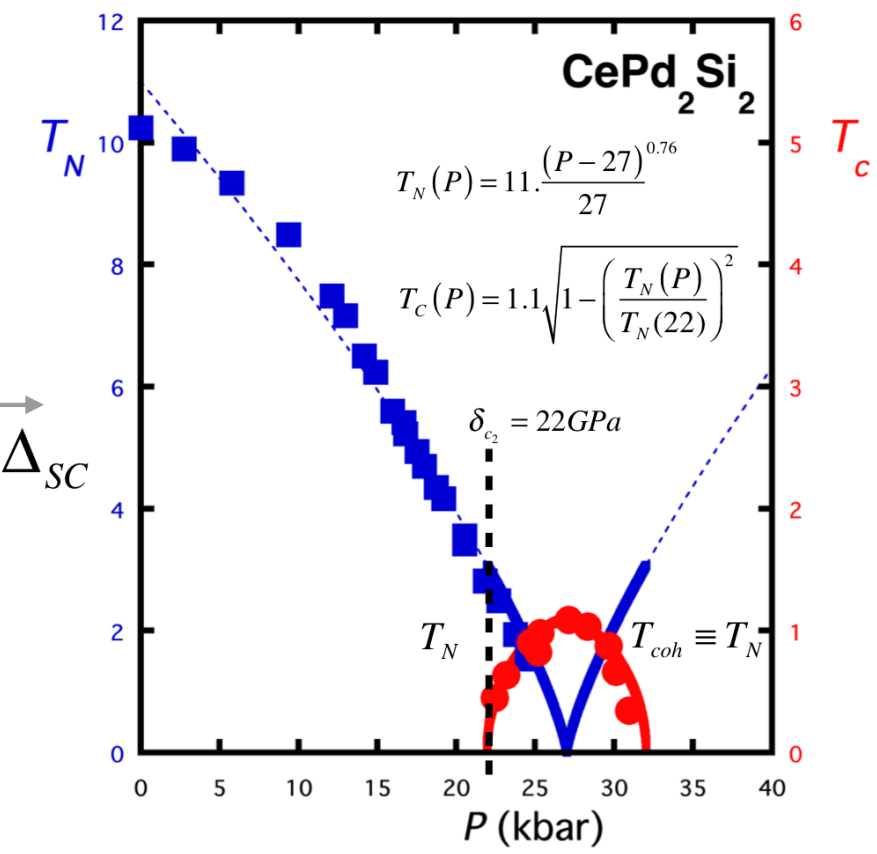

b

\section{Figure 3}

a) Proposed vector rotating in a $\left(\Delta_{O} ; \Delta_{S C}\right)$ plane with an angle determined by $\delta$.

b) Example of application of formula [4], [5] and [6] on $\mathrm{CePd}_{2} \mathrm{Si}_{2}$. The formula for $T_{C}$ automatically uses the fitted value of $T_{N}$ only where there is coexistence between the antiferromagnetic and the superconducting phases (solid curves), although the power law for $T_{N}$ is fitted to all the reported pressure range (dashed curve). For $\delta>\delta_{c}$, the expression for $T_{N}$ corresponds, in fact, to that of the coherence line $T_{c o h}$ as their power laws often have the same exponents in low dimensions. 
[1]P.M Chaikin and T.C. Lubensky, Principles of condensed matter physics, (Cambridge University Press, Cambridge UK) (1995)

[2]S.L. Sondhi, S.M. Girvin, J.P. Carini and D. Shahar, Rev. Mod. Phys.

69(1997)315-333

[3]S.M. Stishov, Phys. Uspekhi 47(2004)789-795

[4] H. von Löhneysen, A. Rosch, M. Vojta, P. Wölfle M P, Rev. Mod. Phys. 79 (2007) 1015-1075

[5]Sachdev S, Quantum Phase Transitions, Cambridge University Press, Cambridge(1999)

[6] Continentino M C, Quantum scaling in many-body physics, World Scientific, Singapore (2001)

[7] K. Levin, D.L. Mills and S.L. Cunningham, Phys. Rev. B10(1974)3821-31

[8] G. Bilbro and W.L. McMillan, Phys. Rev. B14(1976)1887-92

[9] C.A. Balseiro and L.M. Falicov, Phys. Rev. B20 (1979) 4457-4464

[10] M Ido,Y. Okayama, Y. Ijiri and Y. Okajima, Phys. Soc. Jpn. 59(1990)1341-1347

[11] M. Núñez-Regueiro, J-M. Mignot and D. Castello, Europhys. Lett. 18 (1992) 5357

[12] S. Yasuzuka, K. Murata, T. Fujimoto, M. Shimotori and K. Yamaya, J. Phys.

Soc. Jpn. 74 (2005) 1782-1786

[13] R. Jaramillo et al. Nature 459(2009)405-409

[14] S.C. Zhang, Science 275 (1997)1089-1096

[15] E. Demler, W. Hanke and S.C. Zhang, Rev. Mod. Phys. 76(2004)909-974

[16] H.J. Schulz H J (1998), ArXiv:cond-mat/98088167.

[17] R. Fernandes and J. Schmalian, Phys. Rev. B 93(2010) 014521

[18] D. Podolsky, E. Altman, T. Rostunov, and E. Demler E, Phys. Rev. Lett. 93(2004)246402

[19] M. Núñez-Regueiro, Sol. State. Comm. 60, (1986) 797-800

[20] M. Núñez-Regueiro, G. Ortiz, M.D. Núñez-Regueiro , B. Alascio, Physica C153155(1988)245-246

[21] M. Continentino, J. Phys. Soc. Japan 78(2009) 104701

[22] T. Yamauchi and Y. Ueda, Phys. Rev. B77(2008)104529

[23] H. Wilhelm et al., Eur. Phys. J. B21 (2001)175-183

[24] E. Morosan E et al., Nat. Phys. 2 (2006)544-547

[25] S. Nandi et al., Phys. Rev. Lett. 104(2010)057006

[26] C.W. Chu and V. Diatschenko, Phys. Rev. Lett. 41(1978)572-4

[27] C.W. Chu, Phys. Rev. Lett. 33(1974)1283-5 